\title{
Off-Axis Electron Holography for the Quantitative Study of Magnetic Properties of Nanostructures: From the Single Nanomagnet to the Complex Device
}

\author{
C. Gatel ${ }^{1}$, A. Masseboeuf ${ }^{1}$, E. Snoeck ${ }^{1}$, F. Bonnilla ${ }^{2}$, T. Blon ${ }^{2}$, L.-M. Lacroix ${ }^{2}$, A. Meffre ${ }^{2}$, J.F. Einsle ${ }^{3}$, \\ R.M. Bowman ${ }^{3}$, M.A. Bashir ${ }^{4}$ and M. Gubbins ${ }^{4}$ \\ 1. CEMES-CNRS / University of Toulouse, Toulouse, France \\ 2. Laboratoire de Physique et Chimie des Nano-objets (LPCNO), INSA, Toulouse, France \\ 3. Centre for Nanostructured Media, School of Mathematics and Physics, Queen's University Belfast, \\ UK \\ 4. Seagate Technology, 1 Disc Drive, Springtown, UK
}

Electron holography $(\mathrm{EH})$ is a powerful interferometric TEM method particularly efficient for the quantitative studies of local electrostatic and magnetic fields at the nanoscale over a field of view as large as few microns. We applied this method to study the magnetic properties of two very different systems: single $20 \mathrm{~nm}$ Fe nanocubes and a hard-disk drive writer in operando.

Nanomagnets recently attracted considerable interest due to their possible application as building blocks for hard drive disks and permanent magnets or as nanobiological vectors for drug delivery and hyperthermia. Despite theoretical studies, the size-dependence of spin arrangements in single nanomagnets has not yet been evidenced experimentally due to sensitivity limitations of the investigation tools. The single domain limit, corresponding to the critical nanomagnet size separating vortex/single domain configurations, has never been observed although it will dictate the optimized size for applications.

In such small nano-objects, micromagnetic simulations show that the magnetic internal structure changes from single domain (SD) to vortex states as the cube size increases (Fig. 1a). Some years ago, we reported symmetrical vortices, i.e. vortex of $<001>$ axis, in isolated $30 \mathrm{~nm}$ single crystal Fe cubes with a $14 \mathrm{~nm}$ vortex core size [2]. Next, we showed that vortices can also be stabilized in the presence of dipolar interactions thanks to holes in the cubes inducing a pinning of the vortex core [3]. Here we will focus on smaller Fe cubes thanks to a new Hitachi TEM dedicated to EH with an unprecedented spatial resolution of $0.5 \mathrm{~nm}$. Micromagnetic simulations were performed using the $3 \mathrm{D}$ version of the micromagnetic code OOMMF in order to determine the equilibrium magnetic configuration in the cubes with Fe bulk magnetic parameters. We measured single domain (SD) states (flower state) in $20 \mathrm{~nm}$ isolated cubes with a quantitative accordance of the inner magnetic structure between experience and simulations (Fig. 1b, c, d). It means that we experimentally demonstrate the transition between the single domain and the vortex magnetic states. It is in accordance with theoretical predictions of a transition around $23 \mathrm{~nm}$ for perfect Fe cubes [2,4]. Moreover, micromagnetic simulations show that, for cube sizes closed to the transition between SD and $<001>$ vortex states, vortices of $<001>$ and $<111>$ axes are very close in energy (Fig 1a). We will show that some experimental holograms consists in $<111>$ vortex states. In this presentation, we will present an overview of the different magnetic configurations that can be observed and predicted in Fe ferromagnetic nanocubes of variable sizes.

The proliferation in society of mobile devices accessing data via the 'cloud' is imposing a dramatic increase in the amount of information stored on hard disk drives (HDD) used by servers. To meet this request the HDD industry needs to achieve $2 \mathrm{~Tb} / \mathrm{in}^{2}$ densities. This means significantly increased performance from the magnetic pole of the electromagnetic writer in the read/write head of the HDD. Current state-of-art writing implies complex magnetic pole of sub $100 \mathrm{~nm}$ dimensions, in an engineered 
magnetic shields environment, which needs to deliver strong directional magnetic field onto the smallest possible area of the recording media. Current understanding about the behavior, shape and strength of the magnetic field generated by this nanoscale electromagnet arises from indirect methods.

Within a collaboration with Seagate Technology Company, a new set-up combining EH with in-situ electrical biasing was developed for the first time to quantitatively map the magnetic field generated by the writer as a function of applied electrical current up to $+/-60 \mathrm{~mA}$ in real conditions [5]. A specific process of sample preparation was required to extract the active part of the device directly from the production line, without damaging the magnetic shields and the necessary elements for the current injection. The magnetic induction maps obtained by EH for various applied currents have been successfully simulated using micromagnetic simulation using the geometrical parameters of the writer. Our results allow separating the write pole and the shield contributions. The resulting quantified field maps demonstrate the key features requested in magnetic recording, namely a highly directional magnetic field with a low angular spread.

References:

[1] L.-M. Lacroix et al, J. Am. Chem. Soc. 131 (2009), p. 549

[2] E. Snoeck et al, Nano Lett 8 (2008), p. 4293

[3] L.-M. Lacroix et al, Nano Lett. 12 (2012), p. 3245

[4] W. Rave et al, J. Mag. Mag. Mat. 190 (1998), p. 332

[5] J.F. Einsle, et al, NanoResearch, in press (DOI 10.1007/s12274-014-0610-0)

[6] The authors acknowledge the European Union under the Seventh Framework Programme under a contract for an Integrated Infrastructure Initiative Reference 312483-ESTEEM2 and the French National Research Agency under the "Investissement d'Avenir" program reference No. ANR-10-EQPX-38-01". This work is supported by the French national project EMMA (ANR12 BS10 013 01).
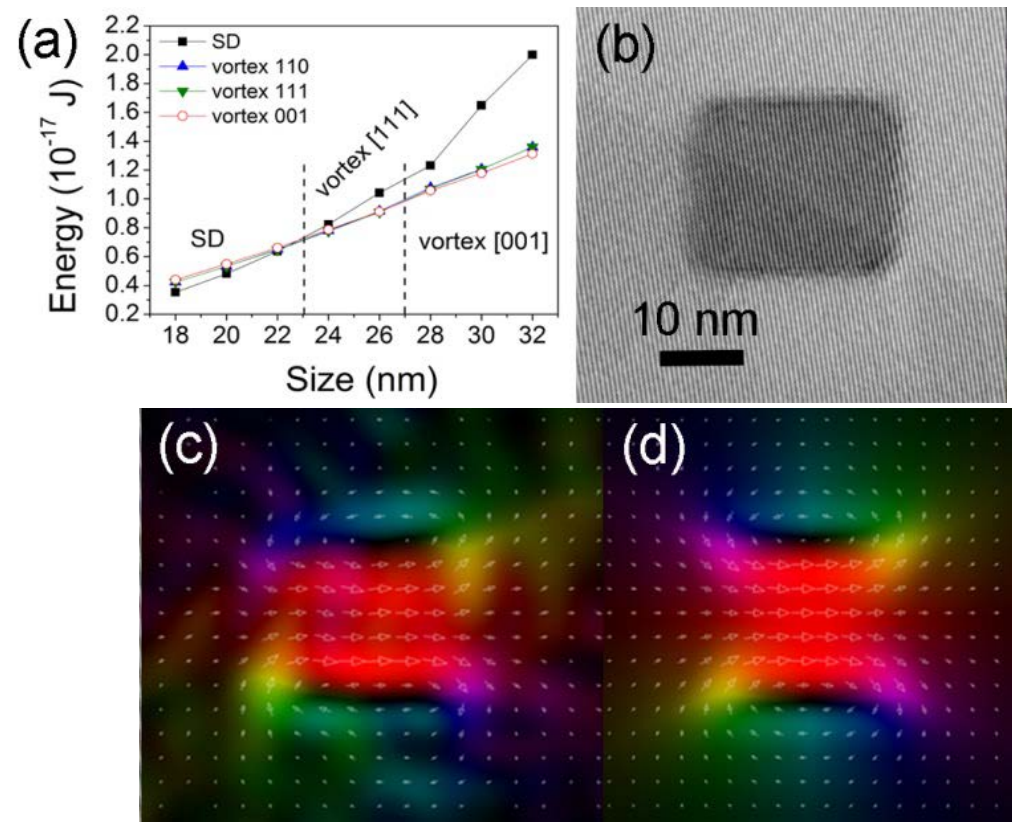

Figure 1. (a) Energies of different magnetic configurations as a function of the $\mathrm{Fe}$ nanocube size (OOMMF calculations). (b) Experimental hologram of a $20 \mathrm{~nm}$ Fe cube. (c) Deduced induction map. (d) Calculated induction map (OOMMF calculations). 